\title{
Quantifying Transient Strain and Energy of Coherent Acoustic Phonons with UEM Imaging
}

\author{
Daniel Du, Daniel Cremons and David Flannigan \\ University of Minnesota, Minneapolis, Minnesota, United States
}

Intense photoexcitation of semiconducting materials produces coherent acoustic phonons, the nature of which renders them amenable to study with ultrafast X-ray and electron scattering [1-3]. For example, coherent acoustic-phonon wavetrains manifest as traveling contrast bands in ultrafast electron microscopy (UEM) image series. In photoexcited Ge, observed time-domain dispersion behaviors of the contrast bands are reminiscent of Lamb-type guided-wave modes. Importantly, this type of phonon excitation is intimately linked to the thermalization of photoexcited charge carriers, with densities above $10^{20} \mathrm{~cm}^{-3}$ resulting in a variety of nonlinear ultrafast effects (e.g., plasma waves and prolonged carrier lifetimes). Indeed, detailed microscopic descriptions of phonon behaviors requires inclusion of multiple effects, including ambipolar diffusion, electron-phonon coupling, and Auger recombination. While modetype can be extracted from phase-velocity and wavenumber dispersion behaviors, the influence of a variety of mechanisms on amplitude - in addition to phonon strain energies - manifest in the contrast-wave strengths in UEM images. Quantitative elucidation of this latter response may enable correlation with charge-carrier effects, in addition to determination of local transient strain energies and profiles.

Here we present calculations of the strain and the strain energies associated with photoexcited coherent acoustic phonons, which give rise to specific transient contrast bands in UEM videos. To accomplish this, direct comparison of normalized image-count amplitudes to calibration curves constructed from simulated first-order symmetric Lamb modes were made. A representative UEM image $(t=300 \mathrm{ps})$ from a typical series is shown in Figure 1a, and the associated averaged normalized image-count difference from a reference image (e.g., $t=0 \mathrm{ps)}$ across a select region of interest (ROI) is shown in Figure 1b. Owing to the complexity of the problem, initial efforts have focused on establishing an upper bound to the strain energies using dynamical electron-scattering models and continuum Lamb-mode distortions [4,5]. By specifically choosing a range of properties to simulate, a series of calibration curves were constructed that linked key characteristics of the Lamb waves to the normalized counts in associated UEM images. In this way, it becomes possible to first associate an observed intensity to a mathematical amplitude of the Lamb wave, as described by the continuum model, and to subsequently connect the amplitude to the desired quantities.

Following the methods outlined above, the maximum strain for each experimentally-observed phonon wavefront returned by the calibration curves corresponding to the position indicated in Figure 1a is plotted in Figure 1c. Interestingly, the wavefronts were found to have similar maximum associated strain values in both the dilatational $x$ and $y$ directions and the shear $x y$ direction across the time range analyzed. While the origins of this anomalous behavior are presently unknown, possible sources may include convoluted multicomponent responses interwoven with specificities of UEM image formation and evolution. Further, the inherent nature of the phonon wavefront renders ascribing a single strain value to a given wave problematic, as the deformation profile itself varies spatially and temporally. Regardless, these methods provide a framework upon which to build a comprehensive model.

Though conventional methods rely on frequency analyses when describing coherent acoustic phonon behaviors, one can pose interesting questions pertaining to the associated transient energies during propagation and decay. Accordingly, volumetric integration of the strain and the associated lattice distortions was performed in order to obtain estimates of the kinetic and potential energies of individual 
wavefronts (Figure 1d). The transient distortions are reported as linear energy densities, denoted by $U$, and are on the order of between 0.5 and $1.0 \mathrm{~nJ} / \mu \mathrm{m}$ for the kinetic response and generally below $0.5 \mathrm{~nJ} / \mu \mathrm{m}$ for the potential energy. For comparison, the optical energy absorbed in the field of view is determined from the optical materials constants, the specimen geometry, and the incident laser-pulse energy $(0.16$ $\mu \mathrm{J} /$ pulse $)$ and spot size $(0.1 \mathrm{~mm})$ to be $1.1 \mathrm{~nJ} /$ pulse. The similar magnitudes suggest that contributions may be made by optical energy outside the field of view to the observed responses, the associated distortion may be overestimated due to additional factors contributing to contrast strength, or that the similarity arises from some combination thereof. Indeed, in light of the experimentally observed $\sim 100$-ps delay between the moment of in situ femtosecond photoexcitation and onset of the first-observed phonon wavefronts in this system [2], charge-carrier thermalization has likely reached completion, and much of the initial energy reservoir has been redistributed throughout the material [6,7].

More exotic explanations aside, it is important to identify and analyze additional sources of specimen motion and experimental specifics that may give rise to confounding effects. Of note are tilting effects, which are known to change the degree to which dynamics and features are visible [8]. Such effects are accounted for by analysis of how tilting influences the image contrast bands. Additional effects to consider include thermal diffuse scattering and Debye-Waller effects, though these contribute an overall modulation of the scattering intensity rather than a local variation in contrast. Such effects are excluded here in order to isolate the phonon responses, though future work will systematically incorporate these mechanisms. As such, a single absorption factor monotonically applied across the specimen was used as a first approximation of absorption in the specimen [4]. This then returns an upper bound to the strains and the energies associated with the phonon wavefronts, as desired [9].
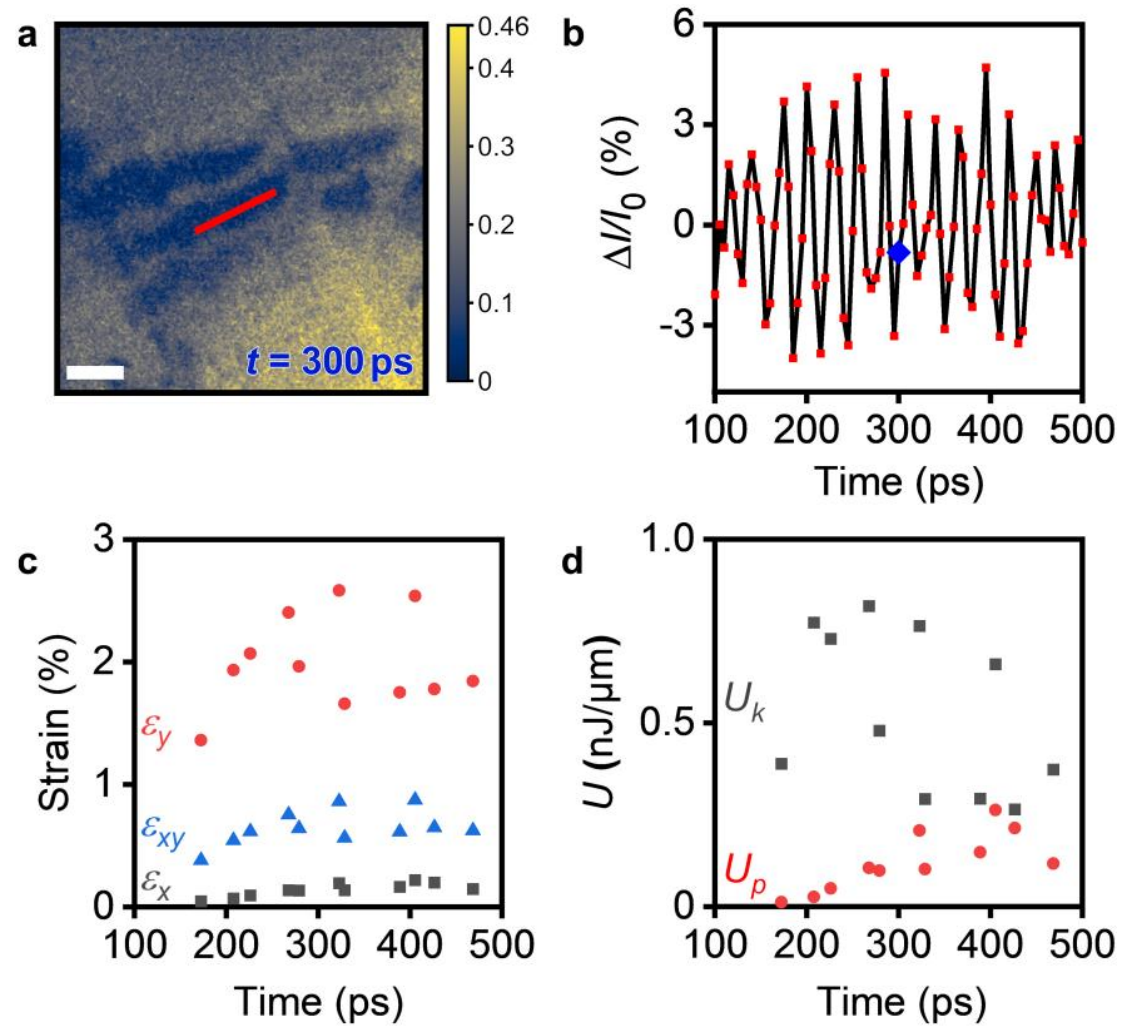

Figure 1. Figure 1. Lamb-mode strain and energy determination from UEM bright-field images. (a) False-colored, representative experimental UEM bright-field image acquired $t=300$ ps after in situ photoexcitation used for simulation purposes. The dark contrast bands are indicative of coherent acoustic 
phonons. The red line marks the ROI from which the intensity data in panel (b) was acquired. The scale bar represents $500 \mathrm{~nm}$. The color bar is counts normalized to vacuum counts. (b) Spatially-averaged normalized difference counts of the ROI shown in panel (a). The $t=300$-ps time point is marked with a blue diamond for reference. (c) Maximum strain (e) in the $\mathrm{x}$ and $\mathrm{y}$ directions, as well as the shear strain (xy), associated with the deformations giving rise to the contrast waves at the ROI in panel (a). (d) Kinetic and potential energies (Uk and Up, respectively) calculated from the maximum strains in panel (c).

\section{References}

[1] A. Cavalleri, et al., Phys. Rev. Lett. 85 (2000), p. 586.

[2] D. R. Cremons, et al., Phys. Rev. Mater. 1 (2017), 073801.

[3] N. V. Chigarev, et al., J. Exp. Theor. Phys. 94 (2002), p. 627.

[4] M. De Graef, Introduction to Conventional Transmission Electron Microscopy (Cambridge University Press, New York, 2003).

[5] D. Royer and E. Dieulesaint, Elastic Waves in Solids, Vol. 1 (Springer, Berlin, 2000).

[6] A. H. Chin, et al., Phys. Rev. Lett. 83 (1999), p. 336.

[7] J. F. Young and H. M. van Driel, Phys. Rev. B 26 (1982), p. 2147.

[8] E. J. VandenBussche and D. J. Flannigan, Ultramicroscopy 196 (2019), p. 111.

[9] This material is based on work supported by the U.S. Department of Energy, Office of Science, Office of Basic Energy Sciences under Award No. DE-SC0018204. This work was supported partially by the National Science Foundation through the University of Minnesota MRSEC under Award No. DMR1420013. We thank Stefano Gonella for discussions pertaining to complex guided-wave phenomena. 